\title{
Analysis on Trust Mechanism of Collaborative Innovation in Supply Chain Enterprises
}

\author{
He Wenzhang \\ Jiujiang University School of Economics and Management Jiujiang City Jiangxi Province
}

Keywords: supply chain; trust mechanism; collaborative innovation

\begin{abstract}
Trust mechanism is essential for collaborative innovation of supply chain enterprise operation, which can reduce operating costs and promote synergy. But in fact, the cooperation relationship between supply chain enterprises is not ideal, which is directly related to mutual distrust among enterprises. In order to improve the level of supply chain management and strengthen the effective establishment of trust relationship such as measurement, recognition and identification, it is particularly important to promote collaborative innovation.
\end{abstract}

\section{Introduction}

Trust means that both sides believe in the other's behavior and belief, with certain asymmetry, infectivity, consistency and empiricism, and also an important reference for the two parties to reach a cooperative relationship. For the collaborative innovation of supply chain enterprises, it is indispensable to select partners rationally, optimize the cooperation standard and strengthen the communication between the two parties, and then consolidate the foundation for strengthening the trust relationship.

\section{The Importance of Trust Mechanism of Collaborative Innovation in Supply Chain Enterprises}

Trust is an important basis for determining the cooperation relationship, and it is also an important reference index for the cooperative innovation operation of supply chain enterprises. It has a positive effect on improving the independent innovation ability of the supply chain members, strengthening the competitiveness of the supply chain, expanding the market share, and so on. It can be seen that the trust is of great significance to the collaborative innovation of the supply chain enterprises.

The relationship between supply chain enterprises, such as principal-agent relationship, cooperative innovation among enterprise members, has the characteristics of adverse selection, and the effective synergy of enterprises is more concerned with the risk of professional ethics among members. The supervision of the supply enterprises should be strengthened to avoid the risk of cooperative innovation, but the supervision is relatively one-sided. It will not only cost a certain amount, but with the deepening of the cooperation relationship, the effectiveness of this supervision will be gradually weakened. Only the mutual dependence, trust and cooperation among the supply chain enterprises can improve the efficiency of cooperation and innovation. At the same time, reduce the cost of operation [1].

The supervision of one party in the supply chain indicates that the distrust of the partner enterprises will increase the supervision cost and also induce the other enterprises to carry out supervision, which seriously affects the establishment and strengthening of the trust relationship and has a negative impact on the collaborative innovation. Only by setting up a good relationship of trust can we promote mutual cooperation among enterprises, and then bring flexibility to synergy. Facing various cooperative risks, the sense of responsibility and win-win situation of the supply chain enterprises is stronger, and it is more conducive to cooperation and innovation among enterprises, thus producing a virtuous cycle [2].

The strong sense of trust in the operation of benign collaborative innovation can help the 
cooperative members to abide by the terms of the contract, avoid the frictions and mistakes of cooperation, and thus reduce the unnecessary negotiation process. The establishment of the trust mechanism can increase the tacit degree of the cooperative enterprise. In the collaborative innovation operation of information exchange and accident, it can provide the risk averse countermeasures for the member enterprises, ignore the defects in the cooperation, avoid the responsibility and the waste of collaborative innovation. And other adverse events. A better understanding and transparency among members will help strengthen the relationship between good collaboration and trust, thereby promoting the flexibility of collaborative innovation and the improvement of resilience.

The supply chain members of the cooperative innovation under the trust mechanism belong to the strategic partners, and the replacement of the partners means that the cooperation innovation and trust mechanism will be restarted, and the cost and risk will be increased, such as the contract and the behavior risk. In this case, the general supply chain enterprises will not rechoose the partners. After a long-term cooperative relationship, it will gradually understand the other's behavior, the contract and behavior risk will also be effectively circumvent, and then promote the mutual benefit of under the strengthening of the trust mechanism[3].

\section{The Establishment of Trust Mechanism of Collaborative Innovation in Supply Chain Enterprises.}

Supply chain enterprises develop synergistic innovation under the trust mechanism, which has a certain process and established mode. Only standardized expansion can strengthen the trust relationship and contribute to collaborative innovation.

Enterprise cooperation can be divided into long-term cooperation and short-term cooperation. Short term cooperation does not exist in common with short-term interests. Long term cooperation is combined with long-term interests and there is a long-term cooperative goal. The cooperative innovation of the supply chain members is a long-term cooperative relationship. There are common interests and goals among the cooperative enterprises. In order to achieve cooperative innovation goals and pursue long-term interests, the cooperation relationship of the supply chain is maintained. When the members of the supply chain cooperate with the target enterprises, there will be no common goals, such as deception and control behavior, which seriously affect the implementation of the trust mechanism among enterprises in the cooperative innovation supply chain, with the promotion of the comprehensive competitiveness of the supply chain and the increasing cooperation risk. It is of great significance to measure and measure the mistrust and reward of member enterprises in collaborative innovation. When it is found that the non standard behavior of the member enterprises has a warning effect on other members' enterprises, it will make the member enterprises take the initiative to abide by the common principles of collaborative innovation, and then improve the perfection of the trust mechanism.

In order to strengthen the trust relationship and improve the effectiveness of collaborative innovation, the supply chain member enterprises in the cooperative innovation operation will predict the future behavior of the trusted members. Prediction is also scientific basis, not imaginary, that is, the continuous collection and analysis of trusted members of the past management ability, trust, and other information, through continuous prediction, verification, can be more aware of the future behavior of trusted members of the enterprise, which for the trust of the members of the enterprise, with the ability to improve behavior and The driving role of continuous learning contributes to collaborative innovation. In order to ensure the smooth operation of collaborative innovation, we need to constantly summarize and adjust the collaborative behaviors among member enterprises. Trust has certain infectivity, distrust and trust will spread and infect in cooperation. But the asymmetry of trust can not be ignored, which depends on the degree of trust between the two parties, and there may be mutual trust between the two parties, and the situation of trust, unworthwhile, and unworthwhile trust of the two parties. In this case, a moderate prediction and evaluation is particularly critical to [4].

Among the members of the supply chain, the ability to predict the future behavior of the other 
party needs to predict the ability of the trusted members to abide by the common principles and carry out their promises, that is, the potential behavior and the ability to overtake the real behavior. This is also the key factor affecting the relationship of trust and cooperation among the members. But the trustworthy image of trust enterprises should be established actively and then infect the trusted enterprises. Based on the supply chain enterprise perspective, trust is the dependence and the degree of trust of the trusted enterprise to comply with the terms of the contract, such as the timely delivery and payment, the long-term high quality, high efficiency service, and the strict implementation of the trust of the contract terms, thus reducing the loss of cooperation conflict and time and resources. Trust is more flexible and transparent than contract restrictions. Partners can negotiate various contract terms and exchange business information at any time in collaborative innovation. The strengthening of the trust mechanism has enhanced the ability to respond to various risks in the supply chain. Through the control of the factors such as human and material resources, the defense ability of the risk events is strengthened. It is also the key to the integration of the supply chain enterprises and the improvement of the rapid response ability.

In the stage of identification, the members of each member will leak their own wishes and ideas, so as to achieve consensus and strengthen the trust relationship, so as to achieve the goal of collaborative innovation. In addition, through communication, the two sides will agree on the significance, value, progress of collaborative innovation, standards and standards that need to be observed, so as to provide guarantee for reducing the friction and contradiction of cooperation.

In the supply chain member enterprises, once the two sides reach a trust relationship, this trust will continue to spread and spread among other enterprises. This trust and cooperation can be divided into two parts, which have a positive effect on the trust mechanism of collaborative innovation in supply chain enterprises, and then provide a trust guarantee for the operation of collaborative innovation. The process of measurement, prediction, recognition, identification and delivery is the process of strengthening trust relationship. Trust and successful experience will enable the two sides to quickly achieve a highly trusted collaborative relationship, namely agile trust. The establishment of agile trust relationship is of great significance to the rapid realization of collaborative innovation goals. The earlier the trust relationship is established, the progress of collaborative innovation activities can be promoted, and its normal operation will be promoted so as to achieve win-win results for the members of the supply chain.

\section{Trust Mechanism of Collaborative Innovation in Supply Chain Enterprises}

There are many factors that affect the cooperation innovation of the members of the supply chain, such as the uncertain factors of the future environmental differences, the speculative factors that the trusted party seeks for the short-term interests, and the control factors that the partners control the cooperative relationship and the unfair distribution of rights, all of which will destroy the cooperation relationship. At the same time, the past experience, cultural belief and behavior attitude of the members of the supply chain are important factors to establish and maintain trust relationship. Zucker represents the mechanism of trust generation, including three categories: process based trust, characteristic trust and normative trust, and the factors that affect the trust mechanism include process factors, characteristic factors and three types of normative factors.

The characteristic trust means that the social background and cultural belief of the cooperative innovation supply chain enterprises are close to each other, the business compatibility is strong, the behavior and the mode of thinking are consistent, and the trust mechanism is more conducive to maintaining the trust mechanism. The factors also involve the similarities between the enterprise culture and the business content and other aspects [4].

Based on the normative trust angle analysis, the establishment of effective trust norms, foreseeable setting of deceit punishment measures to avoid opportunism potential benefits, that is, to improve the cost of fraud prevention and improve the efficiency of cooperation. And the micro normative factors of the loopholes in supply chain enterprises' collaborative innovation. The purpose of the regulation is to reduce speculation and opportunistic interests.

The process based trust angle analysis refers to the mutual cooperative trust based on the 
behavior reliability, continuity and persistence, and the process factors include the periodic communication and information sharing in the collaborative innovation of the supply chain enterprises. It can be seen that the cooperative mechanism in collaborative innovation directly affects the trust degree among the members of the supply chain.

The factors affecting the cooperative innovation of integrated supply chain enterprises can start with the process of collaborative innovation management in supply chain enterprises and establish a cooperative trust promotion mechanism, which is shown as follows.

It is of great significance to standardize the selection of the supply chain members of the collaborative innovation. The reference standard includes the enterprise culture, performance, business organization, and the effectiveness of business capability. The assessment standard includes the speed, quality and development potential of the service, and the assessment object includes the product quality and the production process. Cooperative enterprises are required not only to have comprehensive strength in terms of service and cost, but also to have a standard quality control system and credit level. In order to improve the sense of responsibility, dependence and transparency of the members of the supply chain in cooperative innovation, the characteristics of the partners' business content, the corporate culture and other characteristic factors, as well as the characteristic factors of the two parties, are considered as complementary to each other, and the contradictions, frictions and differences caused by the behavior mode and cultural attitude are reduced as much as possible.

The perfection of cooperation norms can avoid the influence of normative factors on the establishment and continuity of trust mechanism. In this regard, it is particularly important to strengthen institutionalized trust building. The system includes cooperation norms, cooperation clauses, structural constraints, and so on. Institutional trust refers to the incorporation of post punishment measures into the system, forcing the members of the supply chain to abide by common norms in collaborative innovation, and give up the calculation of opportunism to gain interest, which is also called deterrent trust. Based on utilitarian measures, it is necessary to enhance the credibility of both sides. Once the utilitarian arrangement is destroyed, the cost is far greater than opportunistic interests. In this regard, it is of great significance for supplier enterprises to formulate cooperative systems and norms rigorously in collaborative innovation.

First of all, we should set up a multi channel communication channel. Effective communication and communication is the basis for supply chain enterprises to achieve trust and cooperation, and is also a prerequisite for collaborative innovation operation. Effective communication provides a guarantee for information sharing, effective transmission, and mutual trust among partners; in this case, in collaborative innovation, the members of the supply chain can schedule partners online, or face-to-face communication, and regulate partners, such as coordination committees, such as coordination committees, to reduce cooperation. The grinding of the square.

Secondly, the mechanism of knowledge and information sharing is perfected. Knowledge and information sharing is essential for collaborative innovation of supply chain enterprises. Through the transparency of behavior, it can enhance trust and dependence, sense of responsibility, strengthen the trust mechanism and enhance the overall competitiveness of supply chain. Enterprises can train the members of supply chain enterprises to provide quality management, safety management, financial management, marketing skills, strategic planning, inventory management and so on to realize knowledge and information sharing for the members of the supply chain enterprises. The perfection of information knowledge sharing mechanism can realize the consistency of the management mode and concept among the strategic partners, and help the mutual trust between the supply chain enterprises, and thus promote the benign operation of collaborative innovation.

Final moderate dynamic assessment. Collaborative innovation supply chain enterprises, like dynamic networks, strengthen the trust between members by avoiding the influence of corporate culture and communication, such as normative, process and characteristic factors on trust mechanism, but the variability of the influencing factors can not be ignored. 


\section{Conclusion}

As the economic market environment is changing, the supply chain management level is more difficult to break through the bottleneck, which is unfavorable for collaborative innovation operation. In order to meet the customer needs and improve the market share of the supply chain, it is necessary to strengthen the trust mechanism, establish a good cooperative trust relationship, guarantee the cooperative innovation activities, and then improve the comprehensive competitiveness of the supply chain, realize the goal of cooperative innovation quickly, and realize the goal of CO operation.

\section{Acknowledgement}

Foud project: Jiangxi Soft Science Research Project Funding, Project Number: 20161BBA10032 Research on the Collaborative Innovation Mechanism and Capability of New Material Enterprises along the Yangtze River Economic Belt -- Taking Jiujiang as an Example

\section{References}

[1] Fawcett S E, Jones S L, Fawcett A M. Supply chain trust: The catalyst for collaborative innovation [J]. Business Horizons, 2012, 55(2):163-178.

[2] Huang N N. Research on Collaborative Innovation of Supply Chain from the Perspective of Trust [J]. Value Engineering, 2017.

[3] Li Z. Research on the Collaborative Innovation of Supply Chain Enterprises and the Transformation and Upgrading of Regional Economic[C]// International Conference on Smart City and Systems Engineering. IEEE, 2017:273-276.

[4] Wang C, Hu Q. Knowledge sharing in supply chain networks: Effects of collaborative innovation activities and capability on innovation performance[J]. Technovation, 2017. 